\title{
Uncertain DC-DC Zeta Converter Control in Convex Polytope Model Based on LMI Approach
}

\author{
Hafez Sarkawi ${ }^{1}$, Yoshito Ohta ${ }^{2}$ \\ ${ }^{1,2}$ Department of Applied Mathematics and Physics, Kyoto University, Japan \\ ${ }^{1}$ Faculty of Electronics and Computer Engineering, Universiti Teknikal Malaysia Melaka, Malaysia
}

\begin{tabular}{l}
\hline \hline Article Info \\
\hline Article history: \\
Received Dec 21, 2017 \\
Revised Jan 22, 2018 \\
Accepted Feb 7, 2018 \\
\hline
\end{tabular}

\section{Keyword:}

Control duty-ratio

Convex polytope

Dc-dc zeta converter

LMI

LQR

Parameter uncertainty

\begin{abstract}
A dc-dc zeta converter is a switch mode dc-dc converter that can either stepup or step-down dc input voltage. In order to regulate the dc output voltage, a control subsystem needs to be deployed for the dc-dc zeta converter. This paper presents the dc-dc zeta converter control. Unlike conventional dc-dc zeta converter control which produces a controller based on the nominal value model, we propose a convex polytope model of the dc-dc zeta converter which takes into account parameter uncertainty. A linear matrix inequality (LMI) is formulated based on the linear quadratic regulator (LQR) problem to find the state-feedback controller for the convex polytope model. Simulation results are presented to compare the control performance between the conventional LQR and the proposed LMI based controller on the dc-dc zeta converter. Furthermore, the reduction technique of the convex polytope is proposed and its effect is investigated.
\end{abstract}

Copyright $(0) 2018$ Institute of Advanced Engineering and Science. All rights reserved.

\section{Corresponding Author:}

Hafez Sarkawi,

Department of Applied Mathematics and Physics,

Kyoto University,

Yoshidahonmachi, Sakyo-ku, 606-8501 Kyoto, Japan.

Email: hafez@utem.edu.my

\section{INTRODUCTION}

In modern world nowadays, electronic equipment can be found almost everywhere. This electronic equipment usually operates using dc voltage or current. Different electronic equipment normally will use different dc voltage value. In order to produce the desired dc voltage value, a dc-dc converter needs to be deployed. There are several dc-dc converter topologies that can step-up or step-down input voltage namely buck-boost, sepic, cuk, and zeta with various applications [1-5]. Out of these topologies, the later has the least attention given [6] and this motivates the authors to explore this topology for this paper.

Previous research on the dc-dc zeta converter have been presented in [6-16]. To regulate or stabilize the converter, different types of controllers are used such as proportional-integral (PI) [6-9], proportionalintegral-derivative (PID) [10,11], lead compensator [11], adaptive [12-14] and state-feedback [15,16]. In addition, [16] shows the comparison of the performance between PI and state-feedback controllers. Although [6-16] proposed different types of controllers for the dc-dc zeta converter voltage regulation, one thing they share in common that is all of them model their dc-dc zeta converter in nominal value which mean they do not take into account the possible uncertainty in the parameter.

To cope with the uncertainty in the parameter, we employ a convex polytope model for the dc-dc zeta converter. We take into account the parameter uncertainty arising from input voltage and load resistance which are the primary source of uncertainty for the dc-dc zeta converter. Other works [18,19] consider parameter uncertainty in their model but they use other converter topology which is dc-dc boost converter $[18,19]$ and dc-dc buck converter [18]. 
As for the control part of the dc-dc zeta converter, although [15,16] implemented state-feedback control, they use the conventional LQR problem. The conventional LQR approach deals with the optimization of a cost function or performance index. This conventional LQR method produces optimal performance if the system operates in nominal condition. However, when the operating condition change due to uncertainty of the system, the performance deteriorates.

In this paper, we propose LMI based controller design method that can cope with parameter uncertainties in the dc-dc zeta converter. In [17-19], they proposed a design method for state-feedback control based on LMI approach by formulating an LMI for the Hळ fuzzy [17], LQR problem [18], and deriving a new LMI algorithm [19] to find the state-feedback gain. However, the dc-dc- converter is confined to a boost converter $[18,19]$ or a buck converter $[17,18]$. We differentiate our research in two points of view. Firstly, a boost converter can only step-up and a buck converter can only step-down input voltage whereas a zeta converter can operate in both ways. Thus, the control problem for a zeta converter has to deal with large uncertainty caused by the two operations (step-up/step-down). Our contribution is we investigate the dc-dc zeta converter responses in large uncertainty in such a way that there is transition of operation mode from step-down to step-up mode which has never been investigated to the best of the authors' knowledge. Secondly, we investigate the reduction of conservatism in control performance when the system uncertainty is described by a smaller convex polytope. By this way, we can verify the relation of convex polytope reduction with the conservativeness and thus the performance of the controller.

The other sections of this paper are organized as follows. Firstly, in Section 2, we show the modeling of the dc-dc zeta converter under parameters uncertainty. Next, in Section 3, we give LMI based feedback control scheme formulation. After that in Section 4, we present the design examples to illustrate the advantages of this approach. The transient responses have been simulated with PSIM®. Lastly in Section 5, we give the summary.

\section{MODELING OF THE DC-DC ZETA CONVERTER WITH PARAMETERS UNCERTAINTY}

The dc-dc zeta converter circuit is shown in Figure 1 . The circuit consists of two capacitors $\left(C_{l}\right.$ and $\left.C_{2}\right)$, two inductors $\left(L_{l}\right.$ and $\left.L_{2}\right)$, an ideal diode, a dc voltage source $\left(v_{g}\right)$, a resistor $(R)$, and an ideal switch $(S)$. The internal resistances are considered small enough so they can be neglected. The voltage across the capacitors are denoted as $v_{C l}$ and $v_{C 2}$, and the current through the inductors are denoted as $i_{L 1}$ and $i_{L 2}$. The purpose of the circuit is to draw power from the dc voltage source, and supply power to the load at a lower/higher dc voltage value. For our work, it is assumed that the dc-dc zeta converter operates in continuous conduction mode (CCM) all the time.

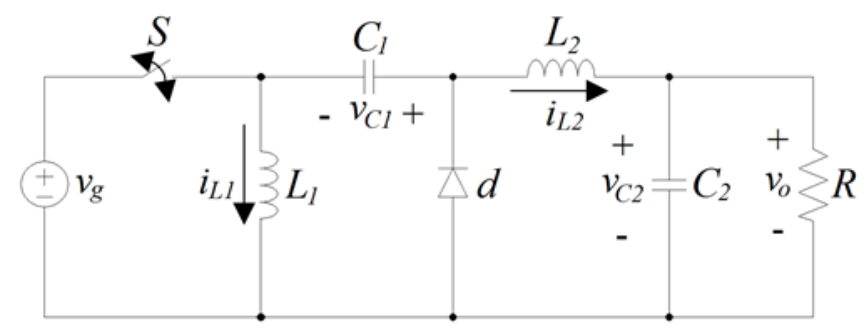

Figure 1. A dc-dc zeta converter circuit

With the switch being closed for the time $d \mathrm{~T}$ and open for $(1-d) \mathrm{T}$, the weighted average of the equation is

$$
\&=\left(A_{o n} d+A_{o f f}(1-d)\right) x+\left(B_{o n} d+B_{o f f}(1-d)\right) v_{g}
$$

where $x$ is the state variables which is $x=\operatorname{col}\left(i_{L 1}, i_{L 2}, v_{C l}, v_{C 2}\right)$ in this paper while $A$ and $B$ are the system and input matrices, respectively. The subscripts on and off refer to the intervals during which the switch is closed and open, respectively. By assuming that the variables are changed around steady-state operating point (linear signal), the variables can now be written as 


$$
\begin{aligned}
& x=X+\tilde{x}, \\
& d=D+\tilde{d}, \\
& v_{g}=V_{g}+\tilde{v}_{g},
\end{aligned}
$$

where $X, D$, and $U$ represent steady-state values, and $\tilde{x}, \tilde{d}$, and $\tilde{v}_{g}$ represent small signal values. During steady-state, the derivatives ( $\&$ ) and the small signal values are zero while for the small signal analysis, the derivatives of the steady-state component are zero. By considering the above conditions and neglecting nonlinear terms, substituting (2) into (1) will produce the small signal averaged model for the dc-dc zeta converter as follows:

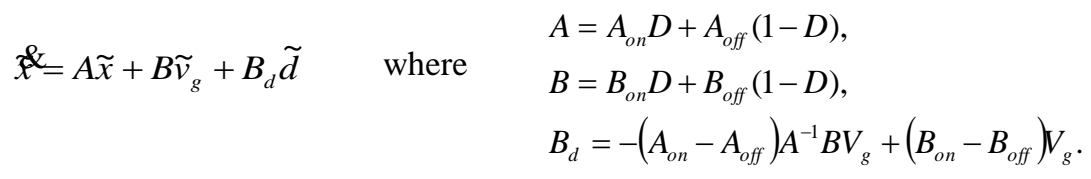

In order to obtain a zero steady-state error between the reference voltage, $V_{\text {ref }}$ and the output voltage, $v_{o}$, the model is augmented with an additional state variable $x_{i n t}$ which stands for the integral of the output voltage error

$$
x_{\text {int }}=-\int\left(V_{r e f}-v_{o}\right) d t \text {. }
$$

The state vector for the new augmented model is now written as $x_{a u g}=\operatorname{col}\left(i_{L 1}, i_{L 2}, v_{C 1}, v_{C 2}, x_{i n t}\right)$ with the matrices defined as follows:

$$
A_{\text {aug }}=\left[\begin{array}{ccccc}
0 & 0 & -\frac{1-D}{L_{1}} & 0 & 0 \\
0 & 0 & \frac{D}{L_{2}} & -\frac{1}{L_{2}} & 0 \\
\frac{1-D}{C_{1}} & -\frac{D}{C_{1}} & 0 & 0 & 0 \\
0 & \frac{1}{C_{2}} & 0 & -\frac{1}{R}\left(\frac{1}{C_{2}}\right) & 0 \\
0 & 0 & 0 & -1 & 0
\end{array}\right], \quad B_{\text {aug }}=\left[\begin{array}{c}
\frac{D}{L_{1}} \\
\frac{D}{L_{2}} \\
0 \\
0 \\
0
\end{array}\right], \quad B_{d(\text { aug })}=\left[\begin{array}{c}
\frac{1}{1-D}\left(\frac{V_{g}}{L_{1}}\right) \\
\frac{1}{1-D}\left(\frac{V_{g}}{L_{2}}\right) \\
\frac{D}{(1-D)^{2}}\left(\frac{1}{R}\right)\left(\frac{V_{g}}{C_{1}}\right) \\
0 \\
0
\end{array}\right] .
$$

The uncertainty for the dc-dc zeta converter is caused when there is variation in the circuit parameters. Since the system and input matrices depend on the uncertain parameters, the new expression of the averaged model for the dc-dc zeta converter without external output can be written as

$$
\mathfrak{f}_{\text {aug }}^{\&}=A_{\text {aug }}(p) \tilde{x}_{\text {aug }}+B_{d(\text { aug })}(p) \tilde{d}
$$

where $p$ represents the uncertain parameters vector defined by $p=\left[p_{1}, \ldots, p_{n}\right]$, where $n_{p}$ is the number of uncertain parameters. The uncertain matrices $A_{a u g}(p)$ and $B_{d(a u g)}(p)$ can be included in a convex polytope, where the set $\left\{G_{l}, \ldots, G_{N}\right\}$ consists of the extrema of the convex polytope which contains the images for all admissible values of $p$

$$
\left[A_{\text {aug }}(p), B_{d(\text { aug })}(p)\right] \in C o\left\{G_{1}, \ldots, G_{N}\right\}:=\left\{\sum_{i=1}^{N} \lambda_{i} G_{i}, \quad \lambda_{i} \geq 0, \quad \sum_{i=1}^{N} \lambda_{i}=1\right\} .
$$

The most significant uncertain parameters for the dc-dc zeta converter are the input voltage $V_{g}$ and the resistive load $R$. Since that output or reference voltage $V_{r e f}$ is constant, the input voltage $V_{g}$ can also be reflected using duty-ratio $D$, which is the one that will be used instead of input voltage $V_{g}$. The inductors and capacitors are not likely to be the candidate because they are not connected to external input/output. In order to describe the convex polytope of the uncertainty, each block must depend linearly on the uncertain parameters. Since $A_{\text {aug }}$ and $B_{d(a u g)}$ matrices have nonlinear terms of the uncertain parameters, two new variables are introduced which are $1 /(1-D)$ and $D /\left((1-D)^{2} R\right)$ to meet with a linear dependence. Thus, the uncertain parameter vector for the dc-dc zeta converter can be written as 


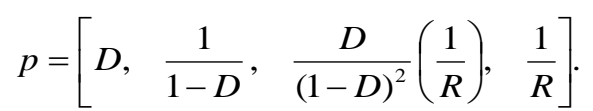

The parameters given by (4) define the uncertain matrices $A_{\text {aug }}$ and $B_{d(a u g)}$, which can be included in the convex polytope (3).

\section{LMI BASED FEEDBACK CONTROL SCHEME FORMULATION}

For a controllable system when all the states are accessible, feedback of all of the states through a gain matrix can be used. The control law used for state-feedback is [20]:

$$
\tilde{u}=K \tilde{x}_{\text {aug }},
$$

where $K$ is the feedback gain matrix.

To optimally control the control effort within performance specifications, a compensator is sought to provide a control effort for input that minimizes a cost function/performance index [21]:

$$
J=\int_{0}^{\infty}\left(\tilde{x}_{\text {aug }}^{T} Q_{w} \tilde{x}_{\text {aug }}+\tilde{u}^{T} R_{w} \tilde{u}\right) d t
$$

where $Q_{w}$ is a symmetric, positive semidefinite matrix and $R_{w}$ is a symmetric, positive definite matrix. By substituting (5) into (6), the cost function can now be written as

$$
J=\int_{0}^{\infty}\left(\tilde{x}_{\text {aug }}^{T}\left(Q_{w}+K^{T} R_{w} K\right) \tilde{x}_{\text {aug }}\right) d t
$$

Using trace operator $\operatorname{Tr}($.$) , the above cost function is equivalent to$

$$
J=\int_{0}^{\infty} \operatorname{Tr}\left(\left(Q_{w}+K^{T} R_{w} K\right) \tilde{x}_{\text {aug }} \tilde{x}_{\text {aug }}^{T}\right) d t=\operatorname{Tr}\left(\left(Q_{w}+K^{T} R_{w} K\right) P_{0}\right),
$$

where $P_{0}=\int_{0}^{\infty}\left(\tilde{x}_{\text {aug }} \tilde{x}_{\text {aug }}^{T}\right) d t$ is a definite positive symmetric matrix that satisfies

$$
\left(A_{\text {aug }}+B_{d(\text { aug })} K\right) P_{0}+P_{0}\left(A_{\text {aug }}+B_{d(\text { aug })} K\right)^{T}+\tilde{x}_{\text {aug }(0)} \tilde{x}_{\text {aug }(0)}^{T}=0
$$

where $\tilde{x}_{\text {aug }(0)}$ represents the initial condition of the state.

Define $P$ by the matrix inequality

$$
\left(A_{\text {aug }}+B_{d(\text { aug })} K\right) P+P\left(A_{\text {aug }}+B_{d(\text { aug })} K\right)^{T}+I<0 .
$$

Because $\left\|\tilde{x}_{\text {aug( }(0)}\right\|<1$, we have

$$
\operatorname{Tr}\left(\left(Q_{w}+K^{T} R_{w} K\right) P_{0}<\operatorname{Tr}\left(\left(Q_{w}+K^{T} R_{w} K\right) P\right.\right.
$$

Hence the matrix $P$ gives an upper bound for the performance of the feedback control (5).

In this paper, it is intended to solve the feedback gain $K$ by minimizing a linear objective function under LMI constraints. Due to the existence of nonlinear term (multiplication of $K$ and $P$ ) in (8), from [22], new variable $Y=K P$ is introduced thus the program can be rewritten as

subject to

$$
\min _{P, Y} \operatorname{Tr}\left(Q_{w} P\right)+\operatorname{Tr}\left(R_{w}^{1 / 2} Y P^{-1} Y^{T} R_{w}^{1 / 2}\right)
$$

$$
A_{\text {aug }} P+P A_{\text {aug }}^{T}+B_{d(\text { aug })} Y+Y^{T} B_{d(\text { aug })}^{T}+I<0
$$

Furthermore, the nonlinear term $\operatorname{Tr}\left(R_{w}^{1 / 2} Y P^{-1} Y^{T} R_{w}^{1 / 2}\right)$ can be replaced by second auxiliary variable $X$

$$
\min _{X} \operatorname{Tr}(X) \text { subject to } X>R_{w}^{1 / 2} Y P^{-1} Y^{T} R_{w}^{1 / 2},
$$


and by using Schur's complement [23]

$$
X>R_{w}^{1 / 2} Y P^{-1} Y^{T} R_{w}^{1 / 2} \leftrightarrow\left[\begin{array}{cc}
X & R_{w}^{1 / 2} Y \\
Y^{T} R_{w}^{1 / 2} & P
\end{array}\right]>0 .
$$

Therefore, the complete LMI formulation of the LQR problem is

$$
\min _{P, Y, X} \operatorname{Tr}\left(Q_{w} P\right)+\operatorname{Tr}(X)
$$

subject to

$$
\begin{gathered}
A_{\text {aug }} P+P A_{\text {aug }}^{T}+B_{d(\text { aug })} Y+Y^{T} B_{d(\text { aug })}^{T}+I<0, \\
{\left[\begin{array}{cc}
X & R_{w}^{1 / 2} Y \\
Y^{T} R_{w}^{1 / 2} & P
\end{array}\right]>0, \quad P>0 .}
\end{gathered}
$$

This LMI formulation can be extended to include the system with uncertain parameter in (4) by replacing the nominal matrices $A_{\text {aug }}$ and $B_{d(\text { aug })}$ in (10) with all the matrices correspond to vertices of the polytope $A_{\text {aug(i) }}$ and $B_{d(\text { aug_i } i)}$ defined in (3) of which can be written as follow

$$
A_{\text {aug(i) }} P+P A_{\text {aug(i) }}^{T}+B_{d\left(\text { aug_i }_{-}\right)} Y+Y^{T} B_{d\left(\text { aug_i }_{-}\right)}^{T}+I<0 .(i=1, \ldots, n)
$$

It is worth to highlight here that the solution of (12) will produce a common matrix $P$ that satisfies (12) at all the vertices of the convex polytope. Once this minimization under constraints is solved, the optimal LQR controller can be recovered by $K=Y P^{-1}$. Since $P$ satisfies (12), this optimal feedback gain $K$ will ensure the quadratic stability of the closed loop system.

\section{DESIGN EXAMPLE}

To see the advantages of our approach, we give a design example in this section. For the dc-dc zeta converter circuit shown in Figure 1, the parameters are tabulated in Table 1. From [15], the input voltage $V_{g}$, the output voltage $V_{o}$ and the duty-ratio $D$ are related by

$$
\frac{V_{o}}{V_{g}}=\frac{D}{1-D}
$$

Equation (13) is the gain (output to input ratio) for the dc-dc zeta converter. For $0<D<0.5$, the converter will step-down the input voltage $v_{g}$ while for $0.5<D<1$, the step-up operation will take place. By solving for $D$ in (13), this would produce $D=0.375$ (for $V_{g}=15 \mathrm{~V}$ ) and $D=0.6\left(V_{g}=6 \mathrm{~V}\right)$. This is one of our research contribution where we investigate the system's response during the transition of operation between step-down $(D=0.375)$ and step-up $(D=0.6)$ operation. In Table 1 , the nominal value $V_{g}=15 \mathrm{~V}$, thus this implies the nominal value for the duty-ratio $D=0.375$. On the other hand, since nominal value for the resistive load is $R=1.5 \Omega$, the corresponding nominal value for the load current is $I_{o}=6 \mathrm{~A}$.

Table 1. The dc-dc zeta converter parameters.

\begin{tabular}{ccc}
\hline Parameter & Value & Nominal value \\
\hline$v_{g}$ & {$[6,15] \mathrm{V}$} & $15 \mathrm{~V}$ \\
$V_{o}\left(V_{\text {ref }}\right)$ & $9 \mathrm{~V}$ & - \\
$R$ & {$[1.5,3] \Omega$} & $1.5 \Omega$ \\
$C_{l} / C_{2}$ & $100 / 200 \mu \mathrm{F}$ & - \\
$L_{1} / L_{2}$ & $100 / 55 \mu \mathrm{H}$ & - \\
$V_{M}$ & $1 \mathrm{~V}$ & - \\
$f$ & $100 \mathrm{kHz}$ & - \\
\hline
\end{tabular}




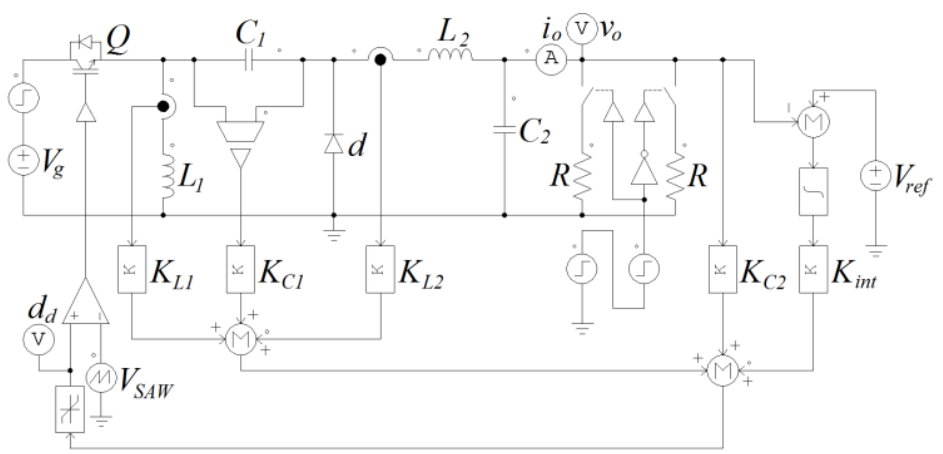

Figure 2. PSIM® simulation diagram for the dc-dc zeta converter with state-feedback controller.
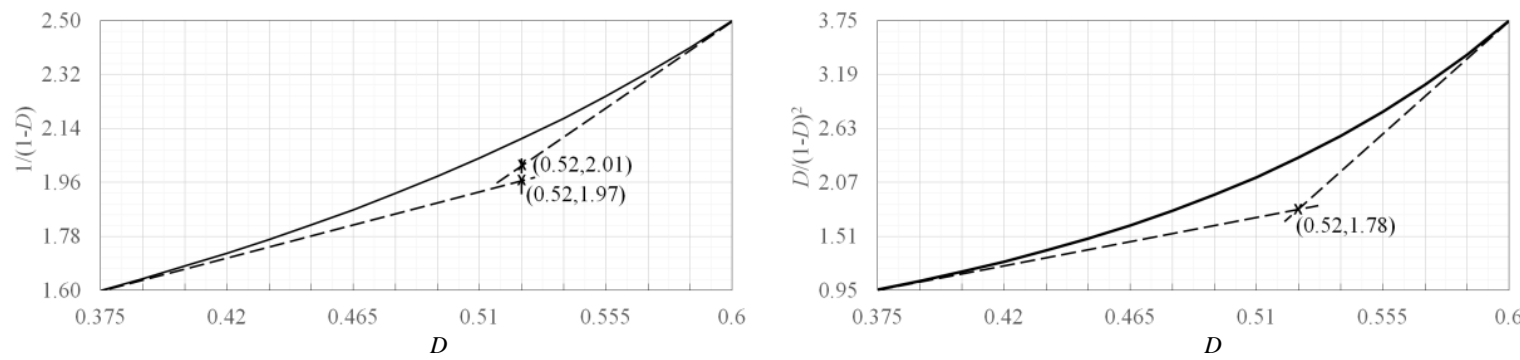

Figure 3. The new coordinates for the uncertain parameters to tighten the convex polytope of uncertainty.

Table 2. The reduced order of uncertain parameter vectors (eight vertices) for the convex polytope of uncertainty.

\begin{aligned} \hline Uncertain parameter vector \\ \hline$p_{1}=[0.375,1.6,0.96 \cdot(0.33), 0.33] \\ p_{2}=[0.375,1.6,0.96 \cdot(0.67), 0.67] \\ p_{3}=[0.52,1.97,1.78 \cdot(0.33), 0.33] \\ p_{4}=[0.52,1.97,1.78 \cdot(0.67), 0.67] \\ p_{5}=[0.52,2.01,1.78 \cdot(0.33), 0.33] \\ p_{6}=[0.52,2.01,1.78 \cdot(0.67), 0.67] \\ p_{7}=[0.6,2.5,3.75 \cdot(0.33), 0.33] \\ p_{8}=[0.6,2.5,3.75 \cdot(0.67), 0.67] \\$\hline\end{aligned}

The simulation diagram for the dc-dc zeta converter is shown in Figure 2. The simulation is done using PSIM $®$ software. As shown in the figure, the resistive load $R$ is varied by means of a voltagecontrolled switch. Voltage and current sensors are deployed to feedback the voltage and current at each capacitor and inductor, respectively. There is a limiter in series with the control duty-ratio line to limit the magnitude to 1 . This is to mimic the actual limit of the comparator.

1. The weight matrices $Q_{w}$ and $R_{w}$ are selected as follows:

$$
Q_{w}=\left[\begin{array}{ccccc}
0 & 0 & 0 & 0 & 0 \\
0 & 1 \cdot 10^{-4} & 0 & 0 & 0 \\
0 & 0 & 0 & 0 & 0 \\
0 & 0 & 0 & 1 \cdot 10^{-4} & 0 \\
0 & 0 & 0 & 0 & 5 \cdot 10^{6}
\end{array}\right], \quad R_{w}=1
$$

2. The criteria for the selection of these weight matrices are such that integral action is enforced and that the control duty-ratio ripple is lower than $20 \%$ of the PWM ramp amplitude $V_{M}$ to avoid nonlinear behavior of the PWM circuitry [24,25].

In this paper, we constructed two convex polytopes of uncertainty for the dc-dc zeta converter. For the first polytope, we constructed them based on hyper-rectangular bounded by minimum and maximum value of the uncertain parameters. There are four uncertain parameters (defined in (4)), thus there is sixteen 
vertices (coordinate) for the first convex polytope of uncertainty. On the other hand, for the second polytope, we proposed a tighter polytope to reduce the conservativeness which is one of the aim of this paper. The procedure for tightening the polytope is depicted in Figure 3. We constructed the tangents of the two extreme points and found the intersection of the tangents. This procedure will produce eight vertices for the convex polytope of uncertainty as shown in Table 2 .

By solving (9), (10), and (11), the conventional LQR controller feedback gain vector $K_{L Q R}$ will be produced, whereas solving (9), (11), and (12) will yield the feedback gain vectors for LMI based controller denoted as $K_{L M I 16}$ (sixteen vertices), and $K_{L M I 8}$ (eight vertices). We use LMI solver in Matlab® [26] called mincx which is based on barrier function of the interior-point method to find $K_{L M I 16}$ and $K_{L M I 8}$. The feedback gain vectors for $K_{L Q R}, K_{L M I 16}$, and $K_{L M I 8}$ are

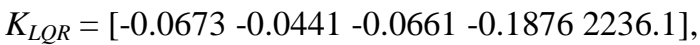

$$
\begin{aligned}
& K_{L M 116}=\left[\begin{array}{lllll}
-0.3755 & -0.0701 & -0.1588 & -0.3408 & 2226.4
\end{array}\right] \text {, }
\end{aligned}
$$

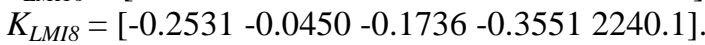

During the nominal condition where $D=0.375$ or $V_{g}=15 \mathrm{~V}$, the responses for the output voltage $v_{o}$ and the load current $i_{o}$ are shown in Figure 4. At this nominal condition, when there is no perturbation, they have nominal $v_{o}=9 \mathrm{~V}$ and $i_{o}=6 \mathrm{~A}$. At $t=0.5 \mathrm{~ms}$, the resistive load $R$ is switched to $3 \Omega$ which is the maximum load allowed in the design, while at $t=3 \mathrm{~ms}$, resistive load $R$ is switched back to $1.5 \Omega$. This correspond to large load current $i_{o}$ deviation of \pm 3 A or equivalent to $\pm 300 \%$. Although there exists large load current perturbation $i_{o}$, all the controllers can regulate the output voltage $v_{o}$. From the output voltage $v_{o}$ figure, either at $t=0.5 \mathrm{~ms}$ or $t=3 \mathrm{~ms}$, out of the three controllers, the $K_{L Q R}$ produces the best performance in term of least overshoot (the magnitude is almost the same with $K_{L M I 8}$ though) and the shortest settling time (assume the system stays at $\pm 5 \%$ of the operating point). As for the comparison between LMI based controllers $K_{L M I 16}$ and $K_{L M I 8}$, the later produces superior performance for both overshoot and settling time. This shows that tightening the convex polytope has reduced the conservativeness and significantly improved the response of the dc-dc zeta converter.

On the other hand, the non-nominal condition occurs when the input voltage $v_{g}$ drop by $60 \%(9 \mathrm{~V})$ to settle at $6 \mathrm{~V}$ as shown in Figure 5. By solving for $D$ in (15), this imply that during non-nominal condition, the duty-ratio $D=0.6$. Figure 6 shows the response during the non-nominal condition. As shown in the figure, at $t=0.5 \mathrm{~ms}$, the $K_{L Q R}$ produces the least overshoot for output voltage $v_{o}$ as compared to the other two controllers but the down side is that the output voltage $v_{o}$ has very high oscillation. Furthermore, at $t=3 \mathrm{~ms}$, its output voltage $v_{o}$ and the load current $i_{o}$ responses deteriorate in such a way that they do no return to their operating point. However, this is not the case for the LMI based controllers. Both the controllers can cope with the large load current perturbation of either $-300 \%$ or $+300 \%$ where the output voltage $v_{o}$ and the load current $i_{o}$ are successfully return to their operating point although the overshoot and settling time are a bit higher compared to those during nominal condition. As expected also, the reduction in the convex polytope produces better controller performance.

We have shown the responses both under nominal and non-nominal condition when subjected to large perturbation. Next, we will show the control duty-ratio response which indicates the control effort that needs to be done to regulate the output voltage of the dc-dc zeta converter. Under the nominal condition (left of Figure 7), the control efforts are quite minimum at both instances ( $t=0.5 \mathrm{~ms}$ and $t=3 \mathrm{~ms}$ ) for all three controllers. As for the non-nominal condition (right of Figure 7), larger control efforts need to be produced at $t=0.5 \mathrm{~ms}$ for the same amount of load current perturbation of $-300 \%$ compared to the nominal condition. The control duty-ratio for the $K_{L Q R}$ deteriorates and at $t=3 \mathrm{~ms}$ where it eventually saturated. On the contrary, for the LMI based controller, both control duty-ratio can return to their operating point.
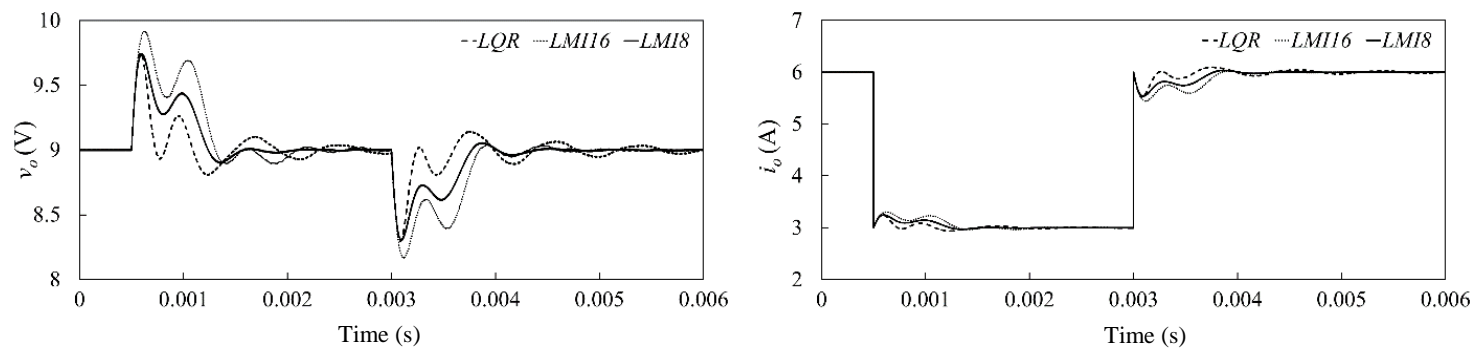

Figure 4. Output voltage (left) and load current (right) responses under nominal condition for $K_{L Q R}$ (dashed line), $K_{L M I 16}\left(\right.$ dotted line) and $K_{L M I 8}$ (solid line). 


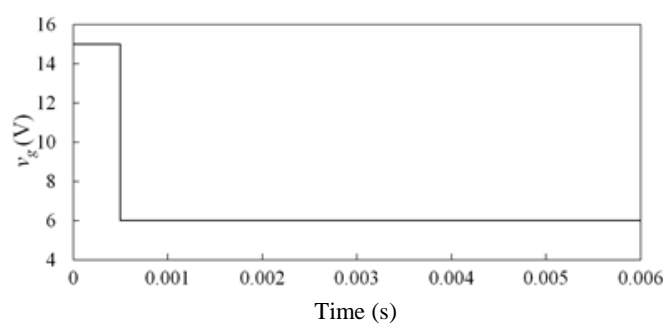

Figure 5. Input voltage perturbation which produces the non-nominal condition.
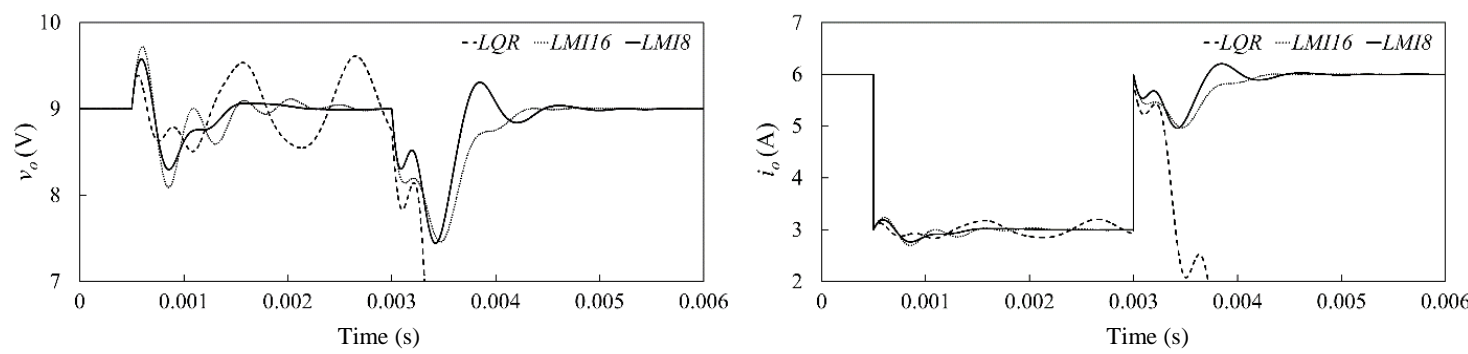

Figure 6. Output voltage (left) and load current (right) responses under non-nominal condition for $K_{L Q R}$ (dashed line), $K_{L M I 16}$ (dotted line) and $K_{L M I 8}$ (solid line).
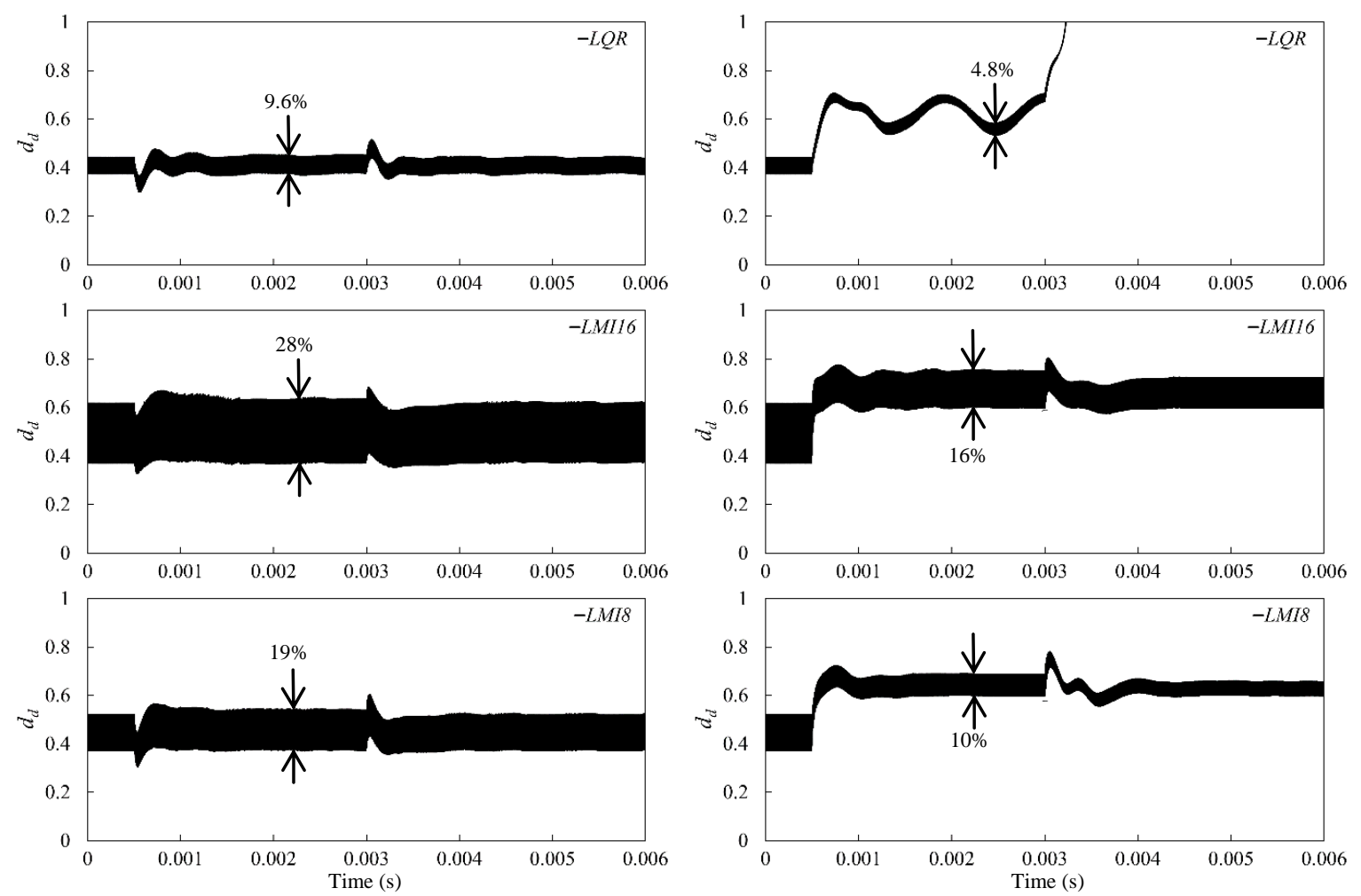

Figure 7. Control duty-ratio response under nominal (left) and non-nominal (right) condition for $K_{L Q R}$ (top), $K_{L M I 16}$ (middle) and $K_{L M I 8}$ (bottom) subject to load current perturbation of \pm 3 A.

The next important criteria that we want investigate is the amount of ripple present in the control duty-ratio signal because certain limit $(<20 \%)$ need to be imposed to avoid nonlinear effect [24,25]. As shown in Figure 7, in both the nominal and the non-nominal conditions, $K_{L Q R}$ propagates the least ripple ( $9.6 \%$ and $4.8 \%$, respectively) followed by $K_{L M I 8}(19 \%$ and $10 \%$, respectively) and the worst ripple is by $K_{L M I 16}(28 \%$ and $16 \%$, respectively) of which the former could poses nonlinear behavior in PWM circuitry if implemented experimentally because it is more than $20 \%$. Since our focus is to prove that the LMI based 
controller is better than the conventional LQR controller especially when the system is highly uncertain, it is worth to mention that by reducing the number of vertices for the convex polytope, it's performances not only improves but also increases the stability margin of the system by reducing the chance of nonlinear behavior in PWM circuitry.

\section{CONCLUSION}

This paper has proposed a dc-dc zeta converter control based on LMI approach by taking account parameter uncertainty. Simulatinn results show that the conventional LQR controller only produces optimal result during the nominal condition whereas the proposed LMI based controller can cope with highly uncertain dc-dc zeta converter. Furthermore, the reduction of the convex polytope not only improves the output voltage response, but also and more crucially reduces the existence of the ripple in the control dutyratio.

\section{REFERENCES}

[1] D. Jayahar, R. Ranihemamalini, and K. Rathnakannan, "Design and Implementation of Single Phase AC-DC BuckBoost Converter for Power Factor Correction and Harmonic Elimination", Int. Journal Pow. Elec. Drive Sys., Vol. 7, No. 3, pp. 1001-1011, Sept. 2016.

[2] S. S. Dash and B. Nayak, "Buck-Boost Control of Four Quadrant Chopper using Symmetrical Impedance Network for Adjustable Speed Drive”, Int. Journal Pow. Elec. Drive Sys., Vol. 5, No. 3, pp. 424-432, Feb. 2015.

[3] D. Saravanan and M. Gopinath, "A Novel Power Factor Correction Modified Bridge Less-CUK Converter for LED Lamp Applications”, Int. Journal Pow. Elec. Drive Sys., Vol. 7, No. 3, pp. 880-891, Sept. 2016.

[4] A. P. C. Rao, Y. P. Obulesh, and C. S. Babu, "Power Factor Correction in Two Leg Inverter Fed BLDC Drive Using Cuk Dc-Dc Converter”, Int. Journal Pow. Elec. Drive Sys., Vol. 6, No. 2, pp. 196-204, June 2015.

[5] M. Oudda and A. Hazzab, "Photovoltaic System with SEPIC Converter Controlled by the Fuzzy Logic", Int. Journal Pow. Elec. Drive Sys., Vol. 7, No. 4, pp. 1283-1293, Dec. 2016.

[6] E. Vuthchhay and C. Bunlaksananusorn, "Modeling and Control of a Zeta Converter", in Proc. IEEE Int. Pow. Elec. Conf., 2010, pp. 612-619.

[7] E. Vuthchhay, C. Bunlaksananusorn, and H. Hirata, "Dynamic Modeling and Control of a Zeta Converter", in Proc. IEEE Int. Sym. on Comm. and Info. Tech., 2008.

[8] M. M. Garg, Y. V. Hote, and M. K. Pathak, "PI Controller Design of a dc-dc Zeta Converter for Specific Phase Margin and Cross-over Frequency", in Proc. IEEE 10th Asian Cont. Conf., 2015, pp. 1-6.

[9] B. Singh, S. Singh, and G. Bhuvanwswari, "Analysis and Design of a Zeta Converter Based Three-Phase Switched Mode Power Supply”, in Proc. IEEE $4^{\text {th }}$ Int. Conf. on Comp. Intel. and Comm. Networks, 2012, pp. 571-575.

[10] R. C. Viero, F. B. D. Reis, and F. S. D. Reis, "Computational Model of the Dynamic Behavior of the Zeta Converter in Discontinuous Conduction Mode", in Proc. IEEE $38^{\text {th }}$ Annual Conf. on Ind. Elec. Society, 2012, pp. 299-303.

[11] R. C. Viero and F. S. D. Reis, "Designing Closed-Loop Controllers Using a Matlab® Dynamic Model of the Zeta Converter in DCM", in Proc. IEEE 10th Int. Conf. on Ind. Apps., 2012, pp. 1-8.

[12] A. Izadian and P. Khayyer, "Complementary adaptive control of Zeta converters", in Proc. IEEE Int. Electric Machines \& Drives Conf., 2013, pp. 1338-1342.

[13] B. Moaveni, H. Abdollahzadeh, and M. Mazoochi, "Adjustable output voltage Zeta converter using neural network adaptive model reference control", in Proc. IEEE $2^{\text {nd }}$ Int. Conf. on Cont., Instr. and Auto., 2011, pp. 552-557.

[14] A. Izadian, P. Khayyer, and H. Yang, "Adaptive voltage tracking control of zeta buck-boost converters", in Proc. IEEE Energy Conv. Cong. and Expo., 2012, pp. 414-417.

[15] H. Sarkawi, M. H. Jali, T. A. Izzuddin, and M. Dahari, "Dynamic Model of Zeta Converter with Full-state Feedback Controller Implementation”, Int. Journal of Research in Eng. and Tech., Vol. 02, No. 08, pp. 34-43, Aug. 2013.

[16] H. Sarkawi and Y. Ohta, "Optimal state-feedback and Proportional-Integral Controller Performance Comparison for Dc-dc Zeta Converter Operating in Continuous Conduction Mode", in Proc. SICE Annual Conf., 2016, pp. 448-451.

[17] N. Kaewpraek and W. Assawinchaichote, " $\mathrm{H}_{\infty}$ Fuzzy State-feedback Control Plus State-Derivative-feedback Control Synthesis for Photovoltaic Systems", Asian Journal of Control, Vol. 18, No. 4, pp. 1441-1452, July 2016.

[18] C. Olala, R. Leyva, A. E. Aroudi, I. Queinnec, "Robust LQR Control for PWM Converters: An LMI Approach", IEEE Trans. Ind. Electron., Vol. 56, No. 7, pp. 2548-2558, July 2009.

[19] C. Olala, I. Queinnec, R. Leyva, A. E. Aroudi, "Optimal State-feedback Control of Bilinear Dc-dc Converters with Guaranteed Regions of Stability”, IEEE Trans. Ind. Electron., Vol. 59, No. 10, pp. 3868-3880, Nov. 2012.

[20] K. Ogata, Modern Control Engineering, $4^{\text {th }}$ Edition, Prentice Hall, 2002.

[21] B. D. O. Anderson and J. B. Moore, Optimal Control: Linear Quadratic Methods, New Edition, Prentice Hall, 1990.

[22] E. Feron, V. Balakrishnan, S. Boyd, and L. El Ghaoui, "Numerical methods for $\mathrm{H}_{2}$ related problems," in Proc. ACC, 1992, pp. 2921-2922.

[23] S. Boyd, L. El Ghaoui, E. Feron, and V. Balakrishnan, Linear Matrix Inequalities in Systems and Control Theory, ser. Studies in Applied and Numerical Mathematics, vol. 15. Philadelphia, PA: SIAM, 1994.

[24] A. El Aroudi, E. Alarcon, E. Rodriguez, R. Leyva, G. Villar, F. Guinjoan, and A. Poveda, "Ripple based index for predicting fast-scale instability of dc-dc converters in CCM and DCM," in Proc. IEEE Ind. Techno. Conf., 2006, pp. 1949-1953. 
[25] E. Rodriguez, G. Villar, F. Guinjoan, A. Poveda, A. El Aroudi, and E. Alarcon, "General-purpose ripple-based fastscale instability prediction in switching power regulators," in Proc. IEEE ISCAS, 2007, pp. 2423-2426.

[26] P. Gahinet, A. Nemirovski, A. J. Laub, and M. Chilali, LMI Control Toolbox for Use with Matlab. Natick, MA: The MathWorks, Inc., 1995.

\section{BIOGRAPHIES OF AUTHORS}

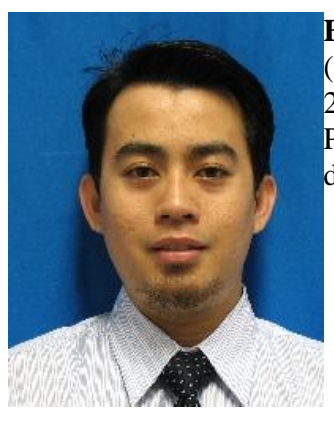

Hafez Sarkawi received his BEng. Electrical (Electronics) from Universiti Teknologi Malaysia (UTM) in 2007 and MEng (Industrial Electronics and Control) from Universiti Malaya (UM) in 2012. Currently he is pursuing his PhD degree at the Department of Applied Mathematics and Physics, Kyoto University, Kyoto, Japan. His research interests inclule modeling and control of dc-dc converter, robust and hybrid control.

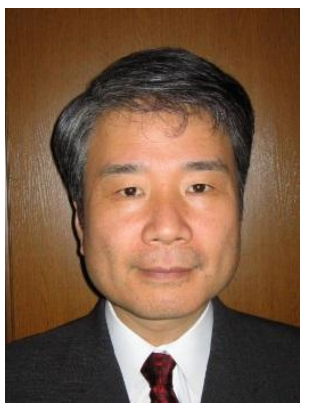

Yoshito Ohta received the Dr.Eng. degree in electronic engineering from Osaka University, Osaka, Japan, in 1986. From 1986 to 1988, he was a Visiting Scientist at the Laboratory for Information and Decision Systems, Massachusetts Institute of Technology. Since 2006, he has been a Professor with the Department of Applied Mathematics and Physics, Kyoto University, Kyoto, Japan. His research interests include modeling of control systems, networked control systems, and robust control. 